\title{
Method of controlling the cyclotron motion of electron beams with a nonadiabatic magnetic field
}

\author{
Alexander Pikin $\odot,{ }^{1,2, *}$ Hannes Pahl $\oplus^{1,3}$ and Fredrik Wenander $\oplus^{1}$ \\ ${ }^{1}$ European Organization for Nuclear Research, Esplanade des Particules 1, 1211 Geneva 23, Switzerland \\ ${ }^{2}$ Centro de Investigaciones Energéticas, Medioambientales y Tecnológicas, \\ Avenida Complutense 40, 28040 Madrid, Spain \\ ${ }^{3}$ Heidelberg Graduate School for Physics, Heidelberg University, \\ Im Neuenheimer Feld 226, 69120 Heidelberg, Germany
}

(Received 1 June 2020; accepted 24 September 2020; published 28 October 2020)

\begin{abstract}
This paper describes a method for reducing the energy of the cyclotron motion for charged particles in coherent beams using a nonadiabatic magnetic field. For the purpose of reducing the cyclotron motion, a local magnetic field reduction of the main guiding field should be situated in the region of the descending phase of the beam oscillation. The required local magnetic field depression can be produced with a soft iron ring or with a magnet coil. The nonadiabatic element can be positioned on the descending part of any period of the beam oscillation where the beam still remains sufficiently coherent. The effect of reducing the cyclotron motion with such nonadiabatic magnetic field is independent of the electric field of the cathodeanode gap and seems to be a universal method for the cyclotron motion control in coherent beams. Therefore, it can be used for reducing the cyclotron motion of electron beams produced with different kinds of guns with different perveances and sizes. For instance, the method is capable of creating ripple-free, laminar beams even for magneto-immersed guns positioned in a magnetic field of only a few hundred Gs and with a cathode emission current density exceeding $30 \mathrm{~A} / \mathrm{cm}^{2}$. It can also be applied for guns producing tubular beams, as demonstrated by our simulations. The results of computer simulations are presented, which demonstrate the capability of effective cyclotron motion control with nonadiabatic magnetic field.
\end{abstract}

DOI: 10.1103/PhysRevAccelBeams.23.103502

\section{INTRODUCTION}

Electron guns with the cathode immersed in the magnetic field (dubbed magneto-immersed electron guns) are attractive because their design is simple, and the electron beams produced with these guns are relatively easy to tune. Producing laminar electron beams with relatively low emission current density from the cathode (like in the electron coolers) can be done with conventional guns by optimizing mostly the electrostatic field of the gun. The task of producing electron beams with high current density requires the use of cathodes with high emission current density positioned in as low magnetic field as possible to reach maximum possible magnetic compression. While the existing cathodes are capable of producing high emission current densities, the cyclotron motion, which is responsible for the electron beam oscillations, limits the minimum

\footnotetext{
*Corresponding author. alexander.pikin@cern.ch

Published by the American Physical Society under the terms of the Creative Commons Attribution 4.0 International license. Further distribution of this work must maintain attribution to the author(s) and the published article's title, journal citation, and DOI.
}

magnetic field for the gun and therefore restricts the maximum magnetic compression of the electron beam. Producing an electron beam with small radial oscillations using a magneto-immersed gun with cathode emission current density over $20 \mathrm{~A} / \mathrm{cm}^{2}$ in a magnetic field of few hundred Gs becomes a serious challenge and is the original goal of this study. We have also extended our investigation to cover the excitation of radial oscillations in an initially laminar electron beam and to the damping of oscillations in tubular beams.

Previous work applying a nonadiabatic (NA) magnetic field, with its extent shorter than the period of the electron cyclotron motion, within the actual cathode-anode gap has demonstrated the possibility of reducing the beam oscillations for some combinations of electron beam parameters [1]. The successful production of laminar, i.e., ripple-free, beams for an emission current density higher than $20 \mathrm{~A} / \mathrm{cm}^{2}$ and for magnetic field in the electron gun region lower than $1 \mathrm{kGs}$, had not been verified. A limiting factor in those earlier studies was the inclusion of the NA element into the gun electrodes. Here, the introduction of a separate NA element grants much more design flexibility and largely decouples the electron gun from the magnetic beam manipulation. 
The NA magnetic field of a cusp geometry has been studied and used extensively for producing axis-encircling electron beams in gyrotron devices [2-6]. Smith [2] analyzed the electron motion in a cusp field and developed an analytical toolbox describing the electron motion in such field. Describing the motion of electrons in rapidly changing magnetic fields is particularly difficult, because their orbital magnetic moment is no longer conserved.

Our motivation was to find a method of generating magnetically confined electron beams with the electron gun positioned in as low magnetic field as possible, thereby attaining as high magnetic compression and beam current density as possible. There are numerous applications that could benefit from such a method for suppressing the cyclotron motion of the beam electrons. They may include electron beam ion sources (EBIS), where laminar high current density electron beams can enable fast charge breeding under stable trap conditions and a range of accelerator applications such as electron lenses and electron coolers. Furthermore, utilizing NA magnetic fields in rf tubes allows one to reduce noise originating from the nonlaminar electron beams $[7,8]$.

Through simulations we have explored the influence of the NA magnetic field on the electron trajectories and on the collective beam behavior. By carefully designing the strength and shape of the NA element the kinetic energy of the transverse motion of the electrons can be reduced by transferring part of it to the axial motion, without violating Busch's theorem. With proper tuning of the NA magnetic field this results in a smooth ripple-free beam. Such a beam necessarily presents an equilibrium of focusing and defocusing forces and its radius is therefore determined by the equilibrium conditions, as given by e.g., Herrmann [9].

\section{SIMULATIONS}

\section{A. Gun geometry and simulation method}

For our simulations we used the two-dimensional simulation suite Trak Charged Particle Toolkit [10]. Most of our presented simulations in this initial study have been performed within a uniform magnetic field $(d B / B<1 E-4)$ to avoid effects associated with adiabatic field gradients. The studied magnetic fields range from 300 to 1000 Gs. Two types of electron guns were used: a Pierce-type gun [Fig. 1(a)] with strong radial electrostatic focusing field in the cathode-anode gap and a gun with anode overlapping the cathode [Fig. 1(b)], which has much smaller radial field than the Pierce gun. The simulated electron guns had cathodes with diameters between 1.7 and $3.0 \mathrm{~mm}$.

\section{B. Uniform magnetic field}

In Ref. [1], the control of the cyclotron motion with an NA element was achieved using an iron ring positioned within the volume of the conical anode for a gun with an

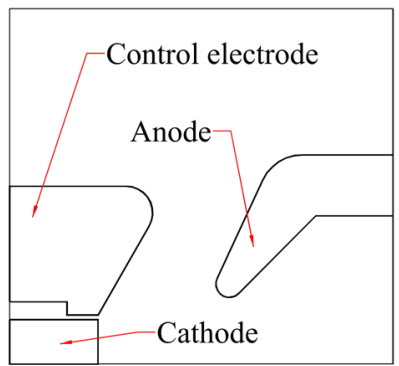

(a)

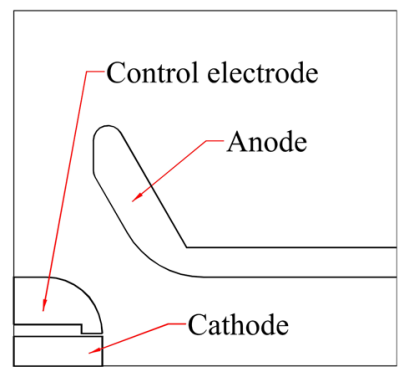

(b)
FIG. 1. Electrostatic models of simulated electron guns: (a) Pierce-type gun and (b) gun with anode overlapping the cathode. The sketches show the profile of the cylindrically symmetric simulation geometries.

electrostatic geometry similar to Fig. 1(b). This approach, however, locks the position of the NA element to the design of the actual electron gun, and therefore limits the parameter range for which the appropriate phase of the cyclotron motion coincides with the location of the NA magnetic field. This fixed position of the NA element did not permit the operation with very small beam oscillations at extreme conditions, i.e., for high current density in combination with a low magnetic field.

By decoupling the position and geometry of the NA magnetic element from the anode, the influence of the NA magnetic field on the cyclotron motion could be optimized freely. We found that if the depression of the magnetic field is produced on the descending phase of the cyclotron motion, the amplitude of the cyclotron motion is reduced. We also found that with careful adjustment of the NA field, the amplitude of the cyclotron motion can in principle be made as small as required. Such adjustment can be achieved by optimizing the geometry of the NA iron ring or by optimizing the geometry and the current if a magnetic coil is used as a NA element.

Figure 2 demonstrates the effect of the NA element on the electron beam oscillations: after the iron ring they practically vanished. One can see that the electron beam equilibrium radius is larger than the cathode radius after the laminar motion is established. The magnetic fluxes through the cathode and the beam in equilibrium are visibly different; this is required to successfully balance the forces caused by the space charge effect. The Herrmann formula yields a beam radius of $1.62 \mathrm{~mm}$, which is in excellent agreement with the observed beam size in the simulation.

A magnet coil can also be used as a NA element, producing a similar cyclotron motion reduction on the oscillating electron beam. Figure 3 presents the result of simulation with the optimized NA magnet coil. The electron beam is generated with an electron gun having the anode overlapping the cathode [as illustrated in Fig. 1(b)].

The effect of a NA magnetic field on the cyclotron motion appears to be largely independent on the electron 


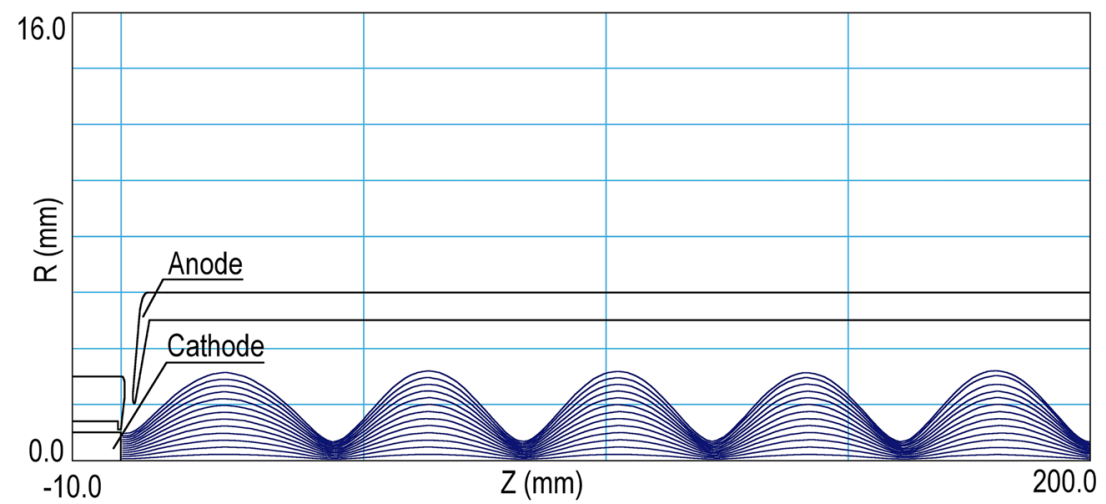

(a)

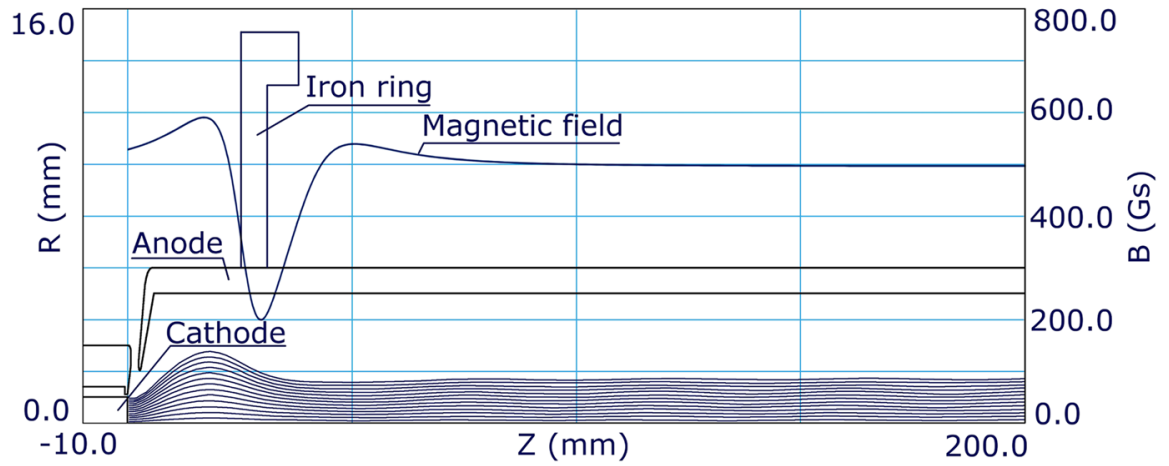

(b)

FIG. 2. Electron beam transmission for $I_{\mathrm{el}}=0.7 \mathrm{~A}$ and $E_{\mathrm{el}}=8.5 \mathrm{kV}$. The electron gun has Pierce geometry with flat cathode, cathode radius $r_{\text {cath }}=1.0 \mathrm{~mm}$ and the emission current density is $j_{e m}=22.3 \mathrm{~A} / \mathrm{cm}^{2}$. (a) No NA element present and a uniform magnetic field $B=500 \mathrm{Gs}$. (b) Iron ring with optimized geometry positioned on the descending phase of the cyclotron motion. The axial distribution of the magnetic field on the beam axis is presented on this plot with the scale on the right.

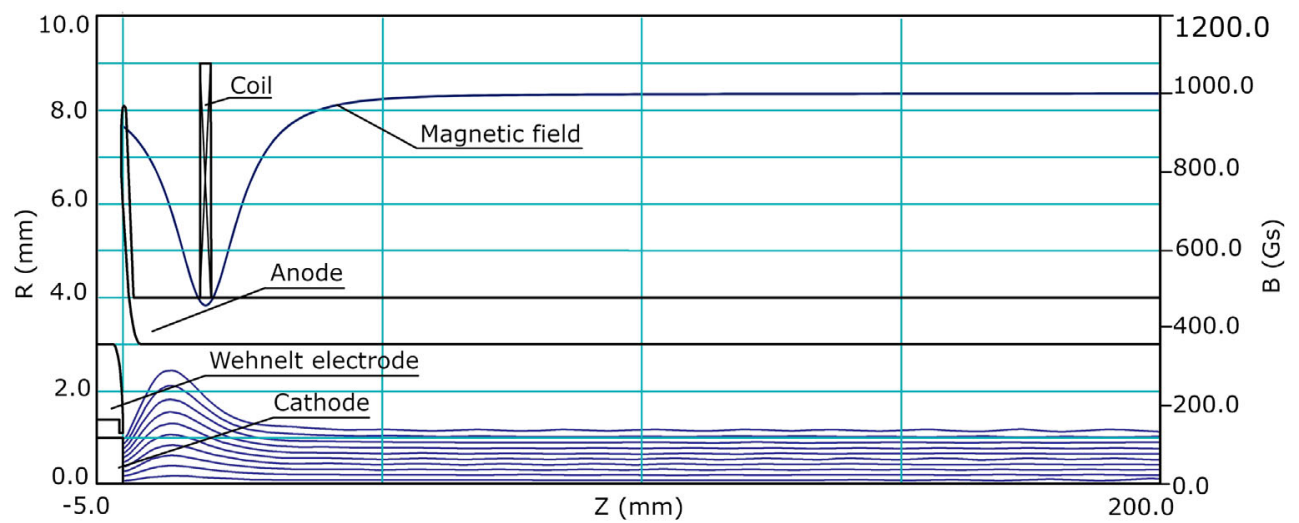

FIG. 3. Cancellation of the cyclotron motion for a beam using a NA coil; $I_{\mathrm{el}}=0.7 \mathrm{~A}, E_{\mathrm{el}}=6.0 \mathrm{kV}, r_{\text {cath }}=1.0 \mathrm{~mm}, B=1.0 \mathrm{kGs}$, and $\mathrm{IN}_{\text {coil }}=850$ Amp-turns (IN-product of coil current I and number of turns $\mathrm{N}$ ).

gun configuration, provided the beam trajectories at different radii are in the same phase.

If the NA element is positioned beyond the electron gun on the second, third or fourth period of the cyclotron motion, it effectively stops the cyclotron motion after the NA element, again leaving only a rigid beam rotation at a constant radius. The electrostatic field of the gun has practically no effect on the electron motion in these cases, confirming that the effect of reducing the transverse momentum should be attributed only to the NA field. Figure 4 illustrates this effect for the NA element being positioned on the third period of the electron cyclotron motion. The observed residual ripples of the electron beam when corrected by a NA element at higher periods 


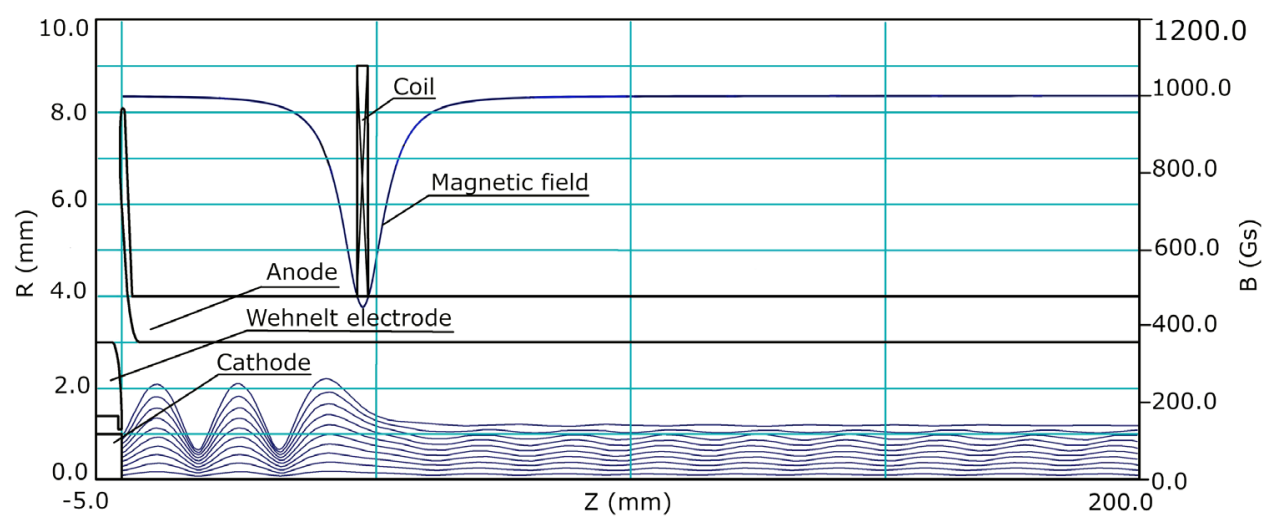

FIG. 4. Effect of a NA magnetic field on the cyclotron motion for a NA coil positioned on the descending part of the third period of cyclotron oscillation: $I_{\mathrm{el}}=0.7 \mathrm{~A}, E_{\mathrm{el}}=6 \mathrm{keV}, r_{\text {cath }}=1 \mathrm{~mm}$, and $B=1000 \mathrm{Gs}$.

can be attributed to some loss of coherency due to shear of the beam layers in electron beams with large space charge density.

\section{Excitation of cyclotron motion}

Similarly, the NA element can excite the cyclotron motion in a laminar electron beam and this excitation can be controllable. Figure 5 shows the beam excitation after it was made laminar with a first NA ring. Such combination of the NA elements allows injection of the highly laminar electron beam into a strong magnetic field and subsequent excitation of the cyclotron motion with the required pitch.

In this case, the cyclotron motion of the passively excited beam starts close to the upstream edge of the NA element, because the decreasing magnetic field breaks the force balance conditions needed for ripple-free beam transport. In the particular case of Fig. 5 both NA iron rings are identical, and the excited beam amplitude is the same as the electron beam originally had before the first NA element. Depending on the strength of the second NA field, the beam excitation can be made different and with required amplitude. NA coils may be preferred over an iron ring for the beam excitation if flexibility in the amplitude control is desired. This method of tailoring the cyclotron motion from a laminar beam may be of interest in the design and operations of radio tubes like e.g., gyrotons.

\section{Injection into increasing magnetic field}

An electron beam with low cyclotron motion, formed with the above described NA element, was tracked into a raising magnetic field with the purpose of producing a high-current density electron beam, which can be applied for EBIS. We used the same Pierce-type electron gun as in Fig. 2 with cathode diameter $2.0 \mathrm{~mm}$, perveances of $0.73 \times 10^{-6} \mathrm{~A} / \mathrm{V}^{1.5}$ and electron current $0.7 \mathrm{~A}$. After optimizing the geometry and the position of the iron ring we obtained an electron beam with very small radial ripple, which propagated smoothly along the adiabatically raising magnetic field of the guiding solenoid. The result of our simulation of this electron beam injection is presented in Fig. 6. With cathode emission current density of $22.3 \mathrm{~A} / \mathrm{cm}^{2}$ the simulated value of the electron beam current density at $17000 \mathrm{Gs}$ is $850 \mathrm{~A} / \mathrm{cm}^{2}$.

This method of cyclotron motion control with a NA field can also be applied for hollow electron beams. For demonstration purposes, a NA magnetic field was applied to a hollow cathode electron gun in a similar fashion as

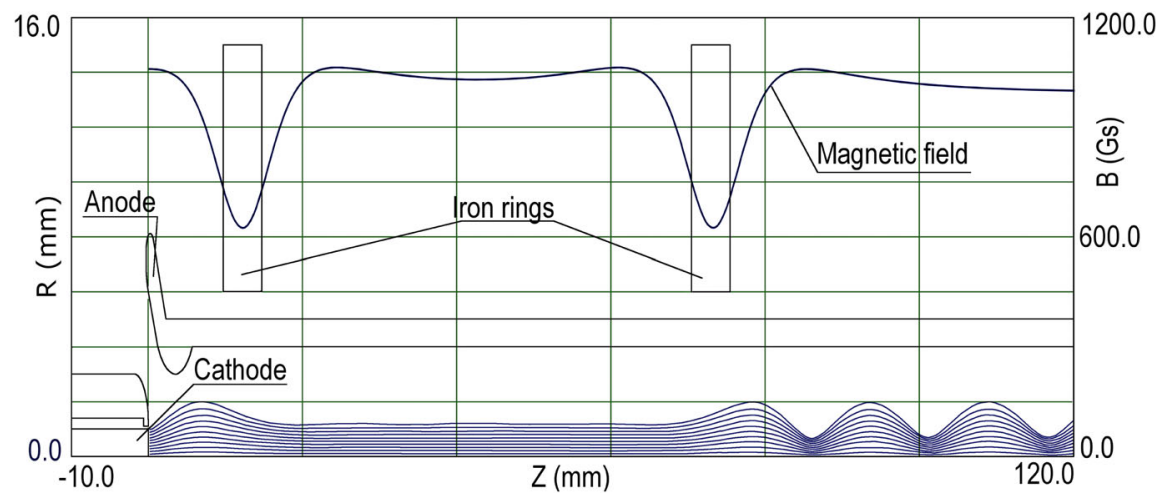

FIG. 5. Excitation of the laminar electron beam with a NA iron ring. $I_{\mathrm{el}}=0.7 \mathrm{~A}, E_{\mathrm{el}}=6.0 \mathrm{keV}, r_{\text {cath }}=1.0 \mathrm{~mm}$, and $B=1.0 \mathrm{kGs}$. 


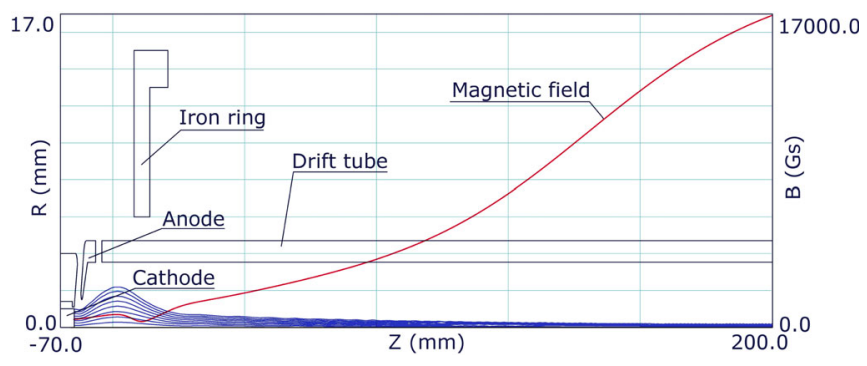

FIG. 6. Injection of the electron beam formed with NA element (iron ring) into a raising magnetic field of the guiding solenoid. $I_{\mathrm{el}}=0.7 \mathrm{~A}, \quad E_{\mathrm{el}}=10 \mathrm{keV}, \quad B_{\text {cath }}=460 \mathrm{Gs}$, and $B_{\text {final }}=17000$ Gs.

described above. The model electron gun with a Pierce geometry had a flat ring cathode with inner radius of $8 \mathrm{~mm}$ and outer radius of $12.5 \mathrm{~mm}$. The cathode is situated in a magnetic field of $480 \mathrm{Gs}$ and after the NA element the beam is compressed adiabatically by the main magnet field increasing up to 2200 Gs. The result of simulation is presented in Fig. 7. As one can see the electron cyclotron motion after the initial bump is significantly suppressed.

\section{E. Analysis of simulation results}

We studied the dependence of the relative amplitude of the beam oscillation $(d r / r)$ on the current in the NA coil for a geometry similar to the one presented in Fig. 3. This analysis has been done with the outermost trajectory of the beam, which has the largest radius and the largest amplitude of the oscillation. Typically, it is considered to represent the beam envelope. This dependence has a pronounced minimum at IN $=860$ Amp-turns, corresponding to $d r / r=0.01$. Both increase and reduction of the coil current from its optimum value results in an increase of the beam oscillation. We also studied dependence of the relative amplitude of the beam oscillation on the axial shift of the coil from its optimized position. Both dependencies are presented in Figs. 8(a) and 8(b). These plots illustrate acceptable deviations of the position of the NA element and its strength for the tolerated relative amplitude of the beam oscillation.

In order to cross-check the particle tracking results, the simulated amplitude of the radial oscillations has been

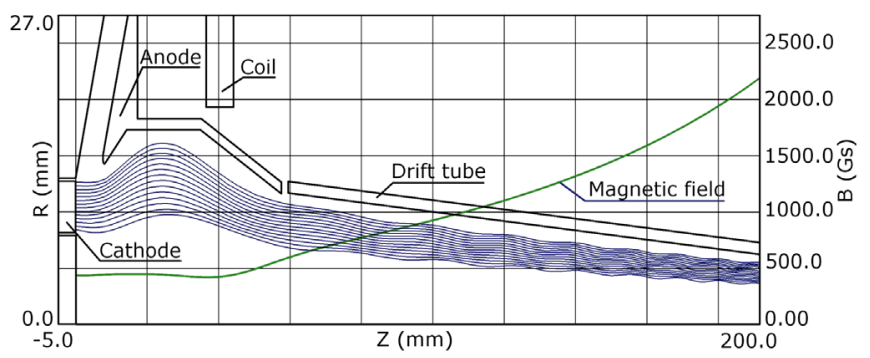

FIG. 7. Hollow electron beam simulation with a NA coil; $I_{\mathrm{el}}=10.0 \mathrm{~A}, E_{\mathrm{el}}=13.7 \mathrm{keV}$, and $B_{\text {cath }}=480 \mathrm{Gs}$.
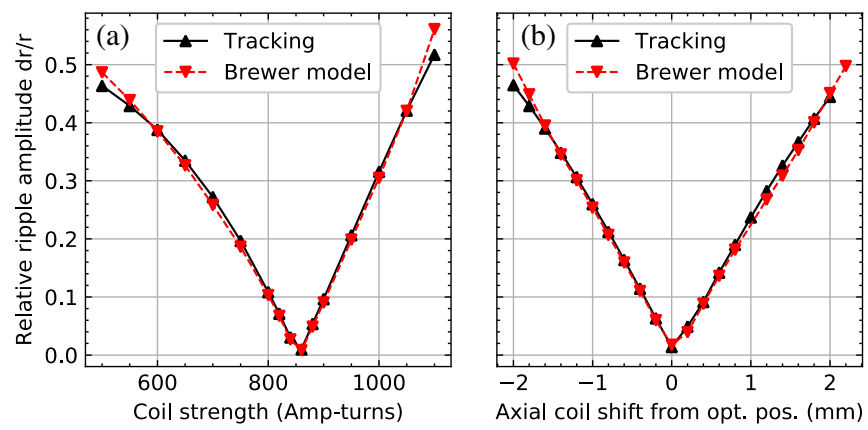

FIG. 8. Sensitivity of relative beam oscillation $d r / r$ on the current in the NA coil (a) and on its axial position (b). The gun and the electron beam parameters are outlined in Fig. 3. The tracking results (black solid) are shown alongside a semitheoretical prediction (red dashed) which is based on an evaluation of the tracking results in a single arbitrary plane downstream of the NA field.

compared to the results of a semitheoretical model. Given a set of initial conditions, the extrema of the radial oscillation can be determined directly from the equation of motion for the beam envelope, as described by Brewer. For this purpose the equation of motion for the radius of a Brillouin beam is partially integrated to obtain a formula for the slope of the beam. The extrema of the radius can then be determined by finding the roots of this expression [11]:

$$
\begin{aligned}
R^{\prime 2}= & \ln \left(\frac{R}{R_{0}}\right)-\left(\frac{\omega_{H}}{\omega_{p}}\right)^{2}\left[R^{2}-R_{0}^{2}+K\left(\frac{1}{R^{2}}-\frac{1}{R_{0}^{2}}\right)\right] \\
& +R_{0}^{\prime 2}=0 .
\end{aligned}
$$

Here, $R=r / r_{m}$ denotes the beam radius normalized by its equilibrium value and $R^{\prime}=d R /\left(d \omega_{p} t\right)$ is the normalized slope; their initial values are given by $R_{0}$ and $R_{0}^{\prime}$ respectively. The magnetic flux ratio between the cathode and the focused beam is parametrized through $K=r_{c}^{4} B_{c}^{2} /\left(r_{m}^{4} B^{2}\right)$. The Larmor frequency and the plasma frequency can be written as $\omega_{H}=e B / 2 m_{e}$ and $\omega_{p}=$ $\sqrt{e I /\left(\pi r_{m}^{2} v_{e} \epsilon_{0}\right)}$, respectively.

The evaluation was carried out at an arbitrary axial position sufficiently far away from any field nonuniformities $(z=100 \mathrm{~mm}$ in Fig. 3). First, the equilibrium radius $r_{m}$ was calculated using the Herrmann formula based solely on the beam current and energy, and the axial magnetic field distribution. Kinetic energy corrections due to the beam space charge were taken into account. This value for the equilibrium radius was subsequently used to determine the dependent quantities like the plasma frequency and the magnetic flux parameter. Second, the beam radius $R_{0}$ and divergence $R_{0}^{\prime}$ were determined from the tracking simulation results by evaluating the outermost trajectory. This is sufficient information to predict the radial extrema and hence the oscillation amplitude downstream with the model 


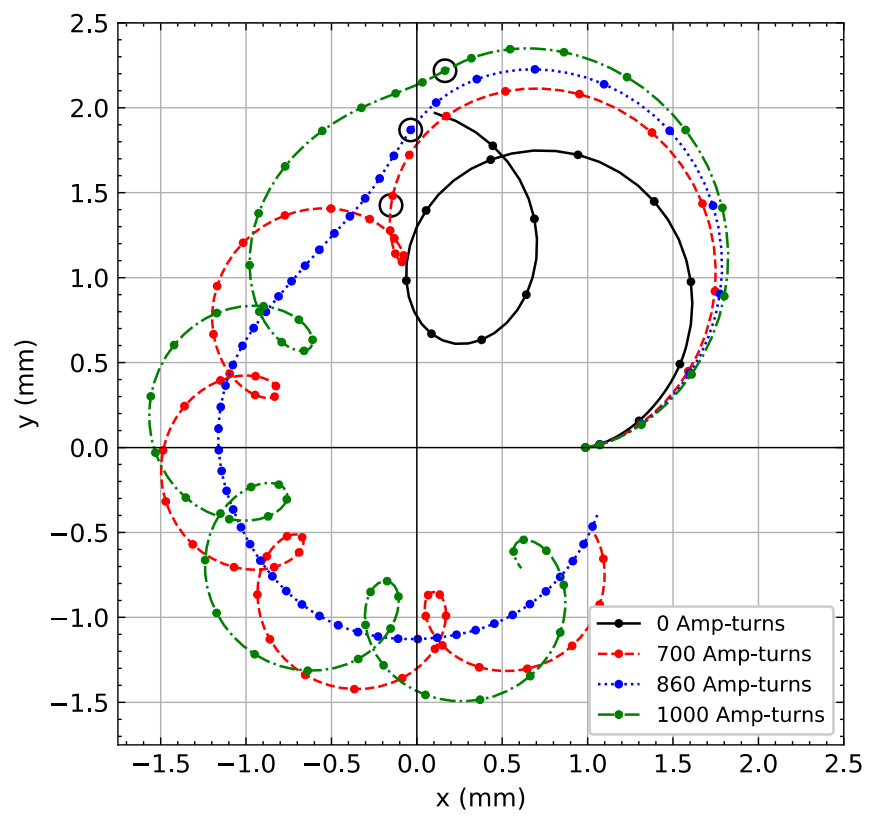

FIG. 9. Transverse motion of the outermost beam electron for the geometry similar to the one presented in Fig. 3. The blue dotted line is for optimized current in the NA coil (860 Ampturns), the red dashed line for insufficient current in the coil (700 Amp-turns), the green dash-dotted line is for excessive current in the coil (1000 Amp-turns), and the black solid line is for zero current in the NA coil. Initially, the particles move counterclockwise. The black circles mark the points where the beams are traversing the minimum of the NA field modulation.

given above. The values obtained in this manner are plotted alongside the simulated ripple amplitude in Fig. 7. The good agreement increases the confidence in the tracking results and suggests that the beam evolution is well described by the existing beam transport equations.

The effect of the cyclotron motion reduction also depends on the geometry of the NA element: its dimensions and shape. Figure 9 shows the transverse motion of the outermost electron in the plane perpendicular to the beam axis for a geometry as presented in Fig. 3. For comparison, the initial part of the track of an electron with zero current in the NA coil is also presented (black solid line).

We can examine the transverse motion of the electron in Fig. 9 for different currents in the NA coil, focusing on the initial part, which includes the emission from the cathode and the first periods of radial oscillation. The markers on the tracks correspond to time points with the same interval of $\Delta t=40 \mathrm{ps}$. A careful examination of the transverse electron motion and the involved magnetic and electrostatic fields shows that the effect of the NA field is complex. If there is no NA element and the energy of azimuthal motion exceeds the value required for the ExB drift, the electron has a strong cyclotron motion component. As the current in the NA coil increases, the resulting reduction of the local magnetic field increases the radius of the azimuthal rotation in the ascending and most of descending part of the electron motion and brings the electrons to larger radius close to the end of its action. On the last crucial portion of the descending part of the electron cyclotron motion the NA field slows down the transverse velocity and changes its direction. One can see that the optimum NA magnetic field sets the electron trajectory on its equilibrium radius with optimum direction where the electron is involved practically only in ExB drift around the beam axis without noticeable cyclotron rotation $(d r / r \approx 1 \%$, blue dotted line). If the NA magnetic field is insufficient, at the end of the descending phase of the cyclotron motion it brings the electron to the radius, which is smaller than the equilibrium radius of the beam and this electron starts its cyclotron motion by moving outward (black solid and red dashed trajectories). For the excessive current in the NA coil the action of the NA field is stronger than needed for the equilibrium between the centrifugal electrostatic and centripetal magnetic force and at the end of its action it brings the electron to the radius, which is larger than is needed for the equilibrium, so the electron moves inwards, starting the cyclotron motion (green dash-dotted trajectory). The situation is somewhat similar to the injection of the electron beam into magnetic field from the gun with electrostatic compression.

\section{CONCLUSION}

We have proposed a method of reducing the cyclotron motion of the electron beam with a NA magnetic field located on the descending part of the cyclotron motion. This method allows controlling the cyclotron motion independently of the electrostatic field of the gun anywhere on the beam path as long as the beam remains coherent and it can be applied to control the cyclotron motion in both solid and hollow beams.

The present paper deals only with a first-order effect of a NA magnetic field on the electron beam, analyzing only a primary beam emitted from the front surface of the cathode. The application of this method for electron beam injection into a raising magnetic field along with the gun design and analysis of such application is planned to be published.

Data is available on request from the authors.

\section{ACKNOWLEDGMENTS}

This research has been done at CERN in the framework of the electron gun development for REXEBIS. $\mathrm{H}$. Pahl has received funding through the Wolfgang Gentner Programme of the German Federal Ministry of Education and Research (Grant No. 05E15CHA).

[1] A. Pikin, J. G. Alessi, E. N. Beebe, D. Raparia, and J. Ritter, Analysis of magnetically immersed electron guns with nonadiabatic fields, Rev. Sci. Instrum. 87, 113303 (2016). 
[2] G. Schmidt, Nonadiabatic particle motion in axial symmetric fields, Phys. Fluids 5, 994 (1962).

[3] J. Sinnis and G. Schmidt, Experimental trajectory analysis of charged particles in a cusped geometry, Phys. Fluids 6 , 841 (1963).

[4] M. J. Rhee and W. W. Destler, Relativistic electron dynamics in a cusped magnetic field, Phys. Fluids 17, 1574 (1974).

[5] W. Hea, C. G. Whyte, E. G. Rafferty, A. W. Cross, A. D. R. Phelps, K. Ronald, A. R. Young, C. W. Robertson, D. C. Speirs, and D. H. Rowlands, Axis-encircling electron beam generation using a smooth magnetic cusp for gyrodevices, Appl. Phys. Lett. 93, 121501 (2008).

[6] C. R. Donaldson, W. He, A. W. Cross, A. D. R. Phelps, F. Li, K. Ronald, C. W. Robertson, C. G. Whyte, A. R. Young, and L. Zhang, Design and numerical optimization of a cusp-gun-based electron beam for millimeter-wave gyro-devices, IEEE Trans. Plasma Sci. 37, 2153 (2009).

[7] Y. Yamaguchia, Y. Tatematsu, T. Saito, R. Ikeda, J. C. Mudiganti, I. Ogawa, and T. Idehara, Formation of a laminar electron flow for $300 \mathrm{GHz}$ high-power pulsed gyrotron, Phys. Plasmas 19, 113113 (2012).

[8] W. M. Manheimer, T. M. Antonsen, Jr., J. P. Calame, B. G. Danly, H. P. Freund, and L. Baruch, Electron and ion noise in microwave tubes, IEEE Trans. Plasma Sci. 31, 32 (2003).

[9] G. Herrmann, Optical theory of thermal velocity effects in cylindrical electron beams, J. Appl. Phys. 29, 127 (1958).

[10] https://www.fieldp.com/trak.html.

[11] G. Brewer, Focusing of Charged Particles, edited by A. Septier (Academic Press, New York, 1967), Vol. 2, Chap. 3.3. 